\title{
A Randomized Pilot Study Comparing the Efficacy of a Therapist- Delivered Motivational Interview to a Brief Computer-Delivered Intervention
}

\author{
Lynn Hernandez ${ }^{1,2}$, Mary Kathryn Cancilliere ${ }^{1}$, Hannah Graves ${ }^{1}$, Anthony Spirito ${ }^{1,3}$ \\ ${ }^{1}$ Brown University, Center for Alcohol and Addiction Studies, Providence, Rhode Island, USA \\ ${ }^{2}$ Brown University, School of Public Health, Department of Behavioral and Social Sciences, Providence, Rhode Island, USA \\ ${ }^{3}$ Brown University, Alpert Medical School, Department of Psychiatry and Human Behavior, Providence, Rhode Island, USA
}

\begin{abstract}
Aims: The purpose of this study was to compare the preliminary efficacy of a computer-assisted intervention (CAI), in which a computer-delivered intervention was immediately followed up with a brief therapist review session, to a therapist-delivered intervention (TDI) for adolescent substance use.

Design, Setting, and Participants: Both conditions were examined in a pilot randomized clinical trial. All participants were recruited from a family court in the northeast United States. The sample included a total of 36 adolescents court-referred for an adolescent substance-related offense.
\end{abstract}

Measures: Measures included adolescent alcohol and marijuana-use frequency, quantity, and problems as well as self-efficacy to resist the urge to use.

Findings: While no significant time-by-condition differences were noted between the CAI and TDI conditions, significant time effects were found for both the TDI and CAI indicating a decrease in the total number of alcohol- or marijuana-use days over the six-month follow-up period.

Conclusions: Given that CAIs are inexpensive, require minimal training, can be implemented with a high degree of fidelity, and are portable when compared to some TDIs, their use for decreasing substance use and related problems, particularly among adolescents with low access to substance-use interventions, seems promising. A fully powered trial of CAI efficacy is indicated.

Motivational interviewing (MI) is a promising therapeutic technique for use with adolescents, given that it: 1) fosters a nonjudgmental and non-confrontational therapeutic environment that acknowledges choices and ambivalence, 2) does not argue against resistance, and 3) supports personal change goals rather than institutional or counselor-based goals (Miller \& Rollnick, 2002). To date, findings from studies conducted with adolescents participating in MI interventions indicate that these interventions are efficacious at reducing alcohol and other drug (AOD) use frequency and related consequences among teens (Bernstein et al., 2010; Goti et al., 2010; Marlatt et al., 1998; Monti et al., 1999; Spirito et al., 2004; Walton et al., 2010; Winters, Fahnhorst, Botzet, Lee, \& Lalone, 2012; Winters, Lee, Botzet, Fahnhorst, \& Nicholson, 2014). Yet qualitative findings indicate that the demand on staff time and training required by MIs can be improved upon (Amodeo et al., 2011).
To circumvent implementation barriers, clinicians and researchers alike have turned to brief, interactive, computerassisted interventions (CAI). CAIs seem promising given that they allow interventions to be delivered at a low cost with minimal training, a high degree of fidelity, and low demand on staff time. These interventions also offer a gamelike appearance, which may appeal to adolescents and easily engage them, thus increasing their acceptability among potentially resistant and hard-to-engage adolescents.

Recently, evidence for the efficacy of these CAIs has begun to emerge. In a review of web-based randomized trials for problematic AOD use by adolescents and young adults, Tait and Christensen (2010) found a small effect size (.22) for alcohol use and a somewhat higher effect (.35) for binge drinking in the 16 studies reviewed. However, of the 16 studies reviewed, only two were with high school students,

Correspondence: Lynn Hernandez, PhD, Brown University School of Public Health, Box GS-121-5, Providence, RI 02912, USA. Phone: 401-863-7688, Email: lynn_hernandez@brown.edu

Financial support: The preparation of this manuscript was made possible from funding from a Career Development Award from the National Institute on Alcohol Abuse and Alcoholism (K01AA021081; PI: Hernandez).

Declaration of interest: The authors declare that they have no competing financial or other interests.

Keywords: computer-delivered interventions, adolescent alcohol and drug use 
and both addressed tobacco use. In a more recent study examining the efficacy of the electronic Check-Up to Go (eCHUG) among ninth graders, results demonstrated that when compared to a standard school drug education control, the e-CHUG lead to reduced positive alcohol expectancies and positive beliefs about alcohol as well as a reduction in drinking frequency and alcohol-related consequences at three-month follow-up (Doumas, Hausheer, Esp, \& Cuffee, 2014).

While results supporting the use of these CAIs seem promising, CAIs have rarely been compared to therapistdelivered interventions (TDI), particularly among adolescents. In a review of 22 studies, Carey, Scott-Sheldon, Elliott, Garey, and Carey (2012) were only able to identify eight studies directly comparing CAIs with TDIs, and all eight studies were conducted among college students, with results favoring the TDI condition. Another study, conducted among urban adolescents visiting an emergency department, compared a standard care condition to a 35minute TDI and a 35-minute CAI with an MI style addressing alcohol misuse and violence (Walton et al., 2010). Participants in both the TDI and CAI demonstrated reductions in alcohol-related consequences at six months when compared to the standard care condition; however, no direct comparisons were made between the TDI and CAI conditions.

Little is known regarding the efficacy of CAIs among adolescents, but the potential cost savings as well as the scalability that is possible with a computerized intervention as opposed to a therapist-delivered intervention make further research on this topic clinically important. Therefore, the purpose of this pilot study was to explore the preliminary efficacy of a CAI and compare it to a brief TDI for reducing AOD use and related risks among court-referred adolescents. We hypothesized that the two intervention conditions would result in comparable effects on AOD use. Both interventions utilized an MI approach, given its developmental appropriateness with adolescents who fail to recognize AOD-related problems and who exhibit low levels of motivation to change. Further, a family-court setting was deemed appropriate given the high co-occurrence of AOD use among court-involved adolescents as well as the limited capacity that exists in such settings to offer AOD-use interventions. Thus, the implementation of computerized interventions within court proceedings increases access to AOD-use care among a population that may otherwise not receive such care.

\section{Method}

\section{Participants}

A total of 42 adolescents referred to the study by the family court system in a northeastern U.S. state for an AOD userelated offense were recruited. Examples of AOD-userelated offenses included possession of alcohol and/or drugs, being at an event where alcohol and/or drugs were present, or being with friends who either possessed or were using alcohol and/or drugs. Study inclusion criteria included participants aged 12 to 18 years old, living at home with at least one parent or guardian, and report of alcohol and/or marijuana use in the prior three months. Exclusion criteria included a substance-dependence diagnosis requiring a higher level of care.

\section{Procedures}

Adolescents were offered the option to take part in the research study or receive the therapist-delivered counseling session but not as part of the research project. Once the referral was received, project staff contacted potential participants and their parents to explain the study and screen for study criteria. Once deemed eligible, adolescents and their parents voluntarily chose to either participate in the study or receive standard counseling. During the first appointment, both written parental consent and adolescent assent were obtained and followed by a baseline assessment battery administered to both parents and adolescents. Adolescents received $\$ 40$ and parents received $\$ 25$ for completing the baseline assessments. Assessments and intervention protocols were completed in a university research setting approximately two miles from the family court. All procedures were approved by the university Human Subjects Protection Office.

After completing the baseline assessment, participants were randomized to either TDI $(n=17)$ or CAI $(n=19)$. Urn randomization procedures, predetermined by the statistician, were used to balance participants by biological sex (male or female) and age. Adolescents who were randomized into the CAI condition and reported alcohol use only or alcohol use plus marijuana use received the alcohol-focused intervention (e-CHUG) while those who reported marijuana use only received the marijuana focused intervention (e-TOKE).

Only adolescent participants were followed up at three and six months after completing the intervention. Follow-up assessments were completed over the phone by research assistants who were blind to study condition. Adolescents received \$20 for completing the three-month follow-up assessment and \$20 for completing the six-month follow-up assessment.

Recruitment and retention. There was a total of 193 adolescents referred by court staff to the project over a 24 month period. Of those referred, 90 reported AOD use during the screening process and were eligible for participation. Of the 90 eligible, 46 agreed to participate. Of those 46 , four participants were participated as pilot subjects to examine feasibility of the intervention, and six adolescents reported at baseline that they had not engaged in AOD use in the prior three months. Therefore, data analyses were conducted on 36 randomized participants. Each AODusing participant in the study completed a one-session TDI $(n=17)$ or CAI $(n=19)$. Follow-up data were available for 15 adolescents in the TDI (88\%) and 18 in the CAI (95\%) at three months, and for 11 adolescents in the TDI $(65 \%)$ and 14 in the CAI (74\%) at six months. 


\section{Conditions}

Therapist-delivered intervention (TDI) condition (for adolescent only). Four masters-level therapists who received MI- and protocol-specific training delivered the TDI. The TDI incorporated procedures central to the MI principles described by Miller and Rollnick (1991). The protocol took between 40 to 60 minutes to complete. Given that most adolescents reported using both alcohol and marijuana, the MI protocol was flexible in addressing the use of both substances but focused primarily on either alcohol or marijuana use based on the adolescent's reported substance of choice and AOD-use patterns.

The MI protocol began with an assessment of the adolescent's level of motivation for change using a decisional balance exercise to evaluate the adolescent's pros and cons for AOD use. The second component included enhancement of motivation to change utilizing individualized normative feedback. This individualized feedback was derived from the baseline assessment and was provided in both written and graphic formats. The feedback form included the adolescent's AOD use along with their perceptions of peer use, and compared these perceptions to national normative information. Information on selfreported negative consequences of AOD use was also presented. The therapist reviewed each of the feedback topic areas with the adolescent, elicited the adolescent's reaction, and provided further information when relevant. The third component included examination of the adolescent's decisional balance and asking adolescents to "envision the future" and think about what positive things might happen if their AOD use were to decrease and what would be the worst thing that could happen if it were to stay the same. This part of the MI was designed to develop the adolescents' sense of discrepancy between current behavior and future goals, and served to increase the adolescents' ambivalence. Finally, in the fourth component of the intervention, the adolescent and therapist collaborated to develop an action plan including identification of goals for behavior change and exploration of barriers to these changes.

Fidelity and competency data for $30 \%$ of the sessions was rated by two coders. Fidelity was rated using a checklist devised for the study that had 14 items covering the essential components of the TDI condition. Ratings for fidelity ranged from $93 \%$ to $100 \%$ with a mean of $96 \%$. For competence ratings, the Motivational Interviewing Treatment Integrity Code Version 3.0 (Moyers, Martin, Manuel, Miller, \& Ernst, 2010), with scores ranging from $1=$ Poor, $3=$ Good, $5=$ Excellent, was used. MI competence ratings ranged from 3 to 5 , which exceeded the expected minimum score of 3 .

Computer-assisted intervention (CAI) condition (for adolescent only). The electronic-Check-Up to Go (eCHUG; http://www.e-chug.com/hs/) and the electronic THC Online Knowledge Experience (e-TOKE; http://www.etoke.com) are managed by the San Diego State University Foundation and were originally designed for college students. However, the developers specifically adapted the original versions for our research team so they would be developmentally appropriate for use with high school students. Examples of components that were adapted include comparison of AOD use patterns and norms to high school students rather than college students, age-appropriate examples of alternatives to money spent on AOD (e.g., cell phone rather than car insurance), and negative consequences to include examples relevant to high school rather than college. Both programs draw from MI principles (Miller \& Rollnick, 2002), social norms feedback (Haines \& Spear, 1996), and self-efficacy and peer modeling (Bandura, 1994) literature. Adolescents in this condition completed either the e-CHUG or e-TOKE based on their substance of choice and their AOD use patterns. Those that reported using both alcohol and marijuana completed the e-TOKE program given that it addresses both alcohol and marijuana use.

On the assessment page of the program, adolescents were asked questions about their alcohol and/or marijuana use quantity, frequency, patterns, and social norms perceptions. Following assessment, the adolescent's responses were computed to provide a personalized feedback report which included their quantity, frequency, and pattern of alcohol and/or marijuana use; amount of time spent under the influence compared to other activities; amount of income spent on alcohol and/or marijuana; national normative comparisons; positive aspects and negative consequences of alcohol and/or marijuana; and assessment of readiness and confidence to make a change. In order to control for time and to make the protocol more comparable to the TDI, therapists met briefly with adolescents to assure that they had completed all components of the e-CHUG/e-TOKE and to complete the decisional balance and action plan worksheets. Therapists spent approximately 10 minutes completing this portion of the CAI with participants.

\section{Measures}

While both parents and adolescents completed baseline measures, for purposes of this study, we only report on adolescent measures and outcomes.

Alcohol and other drug use consumption. Questions from the Adolescent Drinking Questionnaire $(A D Q)$, derived from the Health Behavior Questionnaire (Jessor, Donovan, \& Costa,1991) and the Drug Use Questionnaire (DUQ; Spirito et al, 2001) were used to measure past-three-months drinking and marijuana use frequency. Further, total composite scores for the total number of days that adolescents used either marijuana or alcohol and days adolescents used both alcohol and marijuana simultaneously were also calculated by combining questions from the $A D Q$ and the $D U Q$. Both the $A D Q$ and $D U Q$ were administered at baseline and three- and six-month follow-up assessments.

AOD-use-related problems. The Rutgers Alcohol Problem Index (RAPI) and Rutgers Drug Problem Index (DPI) were used to assess adolescent problem drinking and/or drug use and frequency of related negative consequences (White \& Labouvie, 1989). The RAPI contains 23 questions while the $D P I$ contains 48 questions. Cronbach's alphas for the RAPI were .87 for baseline, .95 for three-month follow-up, and .94 for six-month follow-up. Cronbach's alphas for the DPI were .94 for baseline, .95 for three-month follow-up, and .97 for six-month follow-up. The 10-item Reckless Behavior Questionnaire (RBQ; Shaw, Wagner, Arnett, \& Aber, 1992) 
was used to assess how frequently adolescents engaged in a variety of risk-taking behaviors, including sex without contraception, shoplifting, and destroying public or private property over the previous year.

Self-efficacy. The Brief Situational Confidence Questionnaire (BSCQ; Annis, 1986; Sobell, 1996) was used to examine changes in adolescents' confidence levels as a result of the intervention. The eight items are derived from Marlatt's relapse prevention model (Marlatt \& Gordon, 1985) and include negative emotional states, negative physical states, interpersonal conflict, social problems, and positive emotional states. Responses are provided on 10point Likert scales (i.e., $10 \%$ increments, from $0 \%=$ not at all confident to $100 \%=$ completely confident). Cronbach's alphas for the BSCQ were .87 for baseline, .89 for threemonth follow-up, and .83 for six-month follow-up.

\section{Data Analyses}

Descriptive statistics were used to describe the entire sample. To examine baseline differences, demographic characteristics and baseline assessment scores of the two intervention conditions were compared using chi-square statistics for categorical variables and analysis of variance (ANOVA) for continuous variables. All dependent variables were checked for distributional assumptions. Several had skewed distributions and were log-transformed prior to analysis. Although the small sample size precluded adequate power to test for statistical significance, ANOVAs were used on log-transformed outcome variables to compare the CAI to the TDI on primary AOD-use outcomes ( $A D Q$ and $D U Q)$, secondary substance-related (RAPI and $D P I$ ) outcomes, risky $(R B Q)$ behaviors, and mechanisms of behavior change $(B S C Q)$ at three months and six months.

\section{Results}

Baseline demographics by condition are presented in Table 1. There were no significant differences on the demographic variables, and only one significant difference on the baseline substance-use measures: adolescents randomized to the TDI condition reported greater negative consequences related to drug use on the DPI, $F(1,27)=5.86, p<.05$, than adolescents in the CAI condition.

\section{Table 1}

Baseline Demographics

\begin{tabular}{|c|c|c|c|c|}
\hline \multirow[t]{2}{*}{ Demographics } & \multicolumn{2}{|c|}{$\begin{array}{l}\text { TDI } \\
n=17\end{array}$} & \multicolumn{2}{|c|}{$\begin{array}{l}\text { CAI } \\
n=19\end{array}$} \\
\hline & $n$ & $\%$ & $n$ & $\%$ \\
\hline \multicolumn{5}{|l|}{ Gender } \\
\hline Male & 11 & 64.7 & 9 & 47.4 \\
\hline Female & 6 & 35.3 & 10 & 52.6 \\
\hline \multicolumn{5}{|l|}{ Age } \\
\hline $14-6$ & 7 & 41.1 & 7 & 36.8 \\
\hline $17-18$ & 10 & 58.9 & 12 & 63.2 \\
\hline \multicolumn{5}{|l|}{ Race/Ethnicity } \\
\hline White, non-Hispanic & 12 & 70.6 & 15 & 78.9 \\
\hline Hispanic/Latino & 0 & 0 & 1 & 5.3 \\
\hline African American/Black & 3 & 17.6 & 0 & 0 \\
\hline Other & 2 & 11.8 & 3 & 15.8 \\
\hline \multicolumn{5}{|l|}{ Place of Birth } \\
\hline United States & 11 & 81.3 & 16 & 91.4 \\
\hline Other Country different than U.S. & 6 & 18.8 & 3 & 8.6 \\
\hline \multicolumn{5}{|l|}{ Past 90 Day Use - Yes response } \\
\hline Alcohol & 14 & 82.4 & 17 & 89.5 \\
\hline Marijuana & 17 & 100 & 14 & 73.7 \\
\hline
\end{tabular}

Analysis of AOD-use frequency and AOD-use-related problems, as well reckless behavior, revealed no significant differences across time, with one exception. A significant time effect was observed on marijuana use days, $F(1,26)=$ 13.33, $p<.01$, with participants in both conditions demonstrating significant decreases in their marijuana use from baseline to six months. Results from the time-bycondition repeated measures ANOVA can be seen in Table 2. None of the time-by-condition effects were statistically significant. Effect size estimates for eta squared are also presented in Table 2; all effect sizes were in the small range (Cohen, 1988). 
Table 2

Means, Standard Deviations, and Effect Sizes for Outcome Measures

\begin{tabular}{|c|c|c|c|c|c|c|c|c|c|c|c|c|c|c|}
\hline \multirow{3}{*}{ Measures } & \multicolumn{6}{|c|}{$\begin{array}{l}\text { Therapist-Delivered MI } \\
\text { (TDI) }\end{array}$} & \multicolumn{6}{|c|}{$\begin{array}{l}\text { Computer-Assisted MI } \\
\text { (CAI) }\end{array}$} & \multicolumn{2}{|c|}{$\begin{array}{l}\text { Time } \mathrm{x} \\
\text { Condition }\end{array}$} \\
\hline & \multicolumn{2}{|c|}{$\mathrm{BL}(n=17)$} & \multicolumn{2}{|c|}{$3 \mathrm{M}(n=15)$} & \multicolumn{2}{|c|}{$6 \mathrm{M}(n=11)$} & \multicolumn{2}{|c|}{$\mathrm{BL}(n=19)$} & \multicolumn{2}{|c|}{$3 \mathrm{M}(n=18)$} & \multicolumn{2}{|c|}{$6 \mathrm{M}(n=14)$} & \multirow[b]{2}{*}{$F$} & \multirow[b]{2}{*}{$\eta^{2}$} \\
\hline & $\mathrm{M}$ & SD & $\mathrm{M}$ & SD & M & SD & M & SD & M & SD & M & SD & & \\
\hline \multicolumn{15}{|l|}{ AOD Use } \\
\hline Alcohol Use Days & 6.08 & 8.56 & 17.92 & 23.50 & 15.17 & 18.81 & 17.19 & 24.95 & 13.50 & 16.38 & 10.94 & 16.21 & 1.64 & .06 \\
\hline Marijuana Use Days & 36.25 & 29.34 & 31.75 & 37.72 & 13.50 & 17.90 & 22.10 & 32.87 & 20.06 & 35.42 & 7.31 & 18.74 & .13 & .01 \\
\hline Co-Occurring Use Days & 4.55 & 7.27 & 3.27 & 4.86 & 5.91 & 8.61 & 4.00 & 11.14 & 4.50 & 15.01 & 1.56 & 4.40 & .20 & .01 \\
\hline \multicolumn{15}{|c|}{ AOD-Use-Related Problems } \\
\hline $\begin{array}{l}\text { Alcohol-Related } \\
\text { Problems }\end{array}$ & 10.60 & 5.37 & 14.20 & 14.82 & 18.40 & 17.18 & 8.22 & 6.46 & 5.56 & 5.81 & 6.11 & 6.05 & .29 & .02 \\
\hline Drug-Related Problems & 48.50 & 34.02 & 29.75 & 22.70 & 33.50 & 26.45 & 12.33 & 7.10 & 16.00 & 1.00 & 12.00 & 13.23 & .11 & .02 \\
\hline Reckless Behavior & 7.08 & 3.70 & 6.10 & 5.05 & 5.83 & 5.83 & 5.13 & 3.50 & 3.88 & 3.63 & 2.80 & 3.13 & .32 & .01 \\
\hline \multicolumn{15}{|l|}{ Self-Efficacy } \\
\hline BSCQ & 71.72 & 23.32 & 71.40 & 18.44 & 72.32 & 20.93 & 66.51 & 25.17 & 69.43 & 29.48 & 81.80 & 18.13 & 2.90 & .10 \\
\hline
\end{tabular}

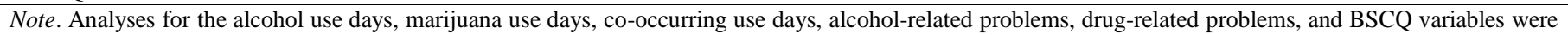
conducted using log-transformed variables; however, the means and standard deviations are reported in their original metric. None of the time-by-condition analyses were statistically significant. Effect size estimates for eta squared recommended by Cohen (1988) are: small, 0.02, medium, 0.13 , and large, 0.25.

The number of participants by condition reporting any alcohol use, marijuana use, and risk-taking behavior (e.g., driving under the influence) across the three time points was also examined. Although not statistically significant, the frequencies demonstrated in Table 3 suggest a similar pattern of reduction in the number of adolescents reporting alcohol or marijuana use over time for the two groups.

Table 3

Frequency of Adolescents Reporting Any Alcohol or Marijuana Use or Driving While Under the Influence

\begin{tabular}{lllllll}
\hline & Baseline & & 3 Months & \multicolumn{2}{c}{6 Months } \\
\cline { 2 - 6 } \multicolumn{1}{c}{ Measure } & TDI & CAI & TDI & CAI & TDI & CAI \\
& $n=17$ & $n=19$ & $n=15$ & $n=18$ & $n=11$ & $n=14$ \\
\hline Alcohol Use & 14 & 17 & 10 & 13 & 7 \\
Marijuana Use & 17 & 14 & 12 & 7 & 6 \\
Driving Under the Influence & 3 & 6 & 0 & 3 & 13 & 1 \\
\hline
\end{tabular}

We were also interested in examining potential mechanisms of change in AOD use, particularly self-efficacy given that this is a variable of main focus in MI. The BSCQ was examined across time and condition; all effects were small and nonsignificant (see Table 2). Although not statistically significant, the frequencies demonstrated in Appendix Figure 1 suggest a pattern of increase in self-confidence to resist the urge to use drugs across eight potential relapse situations as measured by the $B S C Q$.

\section{Discussion}

While CAIs have grown in popularity and shown preliminary efficacy, very little is known about whether they lead to better outcomes than TDIs. Further, most of what is known regarding CAIs is based on studies focusing on college student populations. This study is among the few to compare a CAI to a TDI among a court-referred adolescent population. The study demonstrated feasibility in that adolescents and parents were willing to be randomized to CAI and all participants completed the intervention. Acceptability was indicated by adolescent report that they found the intervention both engaging and useful.
Although there were no statistical differences across intervention conditions, the total number of adolescents reporting use of both alcohol and marijuana at post assessments decreased for both conditions. The findings of this study provide preliminary support for the testing of CAIs in a larger trial because they appear to function comparably to TDIs. The pattern of change illustrated in the CAI compared to the TDI in adolescents' self-efficacy to resist the urge to use substances is also encouraging. Given that CAIs are inexpensive, require minimal training, can be implemented with a high degree of fidelity, and are portable when compared to some TDIs, their use for decreasing AOD use and related problems, particularly among adolescents with low access to substance-use interventions and low motivation to seek substance-use interventions, seems promising and worth fully testing.

Findings from this study should be interpreted in light of several important limitations. First, given that this was an exploratory, pilot study, there were a small number of adolescents in each condition, which affects the stability of these findings and the ability to detect significant differences. In addition, we did not collect data on fidelity and competency of the TDI sessions. Second, given that adolescents in our CAI condition met with a therapist after 
completing the e-CHUG/e-TOKE programs to fill out a decisional balance and action plan worksheet, this pilot study was not a true comparison of a strictly computerdelivered intervention to a therapist-delivered intervention. We choose this design in order to make the CAI comparable to the TDI and to control for time. However, more recently, the e-CHUG/e-TOKE programs were updated to resemble an MI session, with the inclusion of videos giving information on blood alcohol concentration and standard drink measurements, and with the inclusion of questions regarding the adolescent's expectancies, potential alternate behaviors, future goals, and their importance and confidence levels to change. Therefore, the e-CHUG/eTOKE programs now more closely resemble a typical therapist-delivered motivational intervention. Finally, our sample consisted of adolescents referred by the family court system for an AOD-related offense. Therefore, there may have been variability in adolescents' level of use, with some adolescents in our sample either being low-level AOD users or having been "caught" by law enforcement during their first AOD-use experience. Because of the small sample size in this pilot, we were not able to balance the sample on this factor. Thus, the possibility exists that family court involvement alone was sufficient to result in alcohol or marijuana use cessation. There were also other potentially important factors that were not balanced across conditions, such as marijuana use at baseline, where rates appear slightly higher, although not statistically significant, in TDI than CAI.

This study is among the few to have compared the efficacy of a CAI to a TDI among an adolescent sample and to lend preliminary support for the use of inexpensive and easy-toimplement CAI modalities with greater reach given its comparable results to a TDI. A future larger study is needed to test if CAI and TDI are truly comparable as well as moderators of treatment outcome that may indicate differential effectiveness based on adolescent characteristics, such as severity of AOD use, or history of negative consequences due to drug use, which might increase motivation to change. A fully powered trial should also compare the efficacy of these two intervention delivery approaches to standard court proceedings.

\section{References}

Amodeo, M., Lundgren, L., Cohen, A., Rose, D., Chassler, D., Beltrame, C., \& D'Ippolito, M. (2011). Barriers to implementing evidence-based practices in addiction treatment programs: Comparing staff reports on motivational interviewing, adolescent community reinforcement approach, assertive community treatment, and cognitive-behavioral therapy. Evaluation and Program Planning, 34(4), 382-389.

Annis, H. M. (1986). A relapse prevention model for treatment of alcoholics. In W. R. Miller \& N. Heather (Eds.), Treating Addictive Behaviors: Processes of Change (pp. 407-433). New York: Plenum.

Bandura, A. (1994). Regulative function of perceived selfefficacy. In M. G. Rumsey, C. B. Walker, \& J. H. Harris
(Eds.), Personnel selection and classification (pp. 261271). Hillsdale, NJ, Lawrence Erlbaum Associates, Inc. Bernstein, J., Heeren, T., Edward, E., Dorfman, D., Bliss, C., Winter, M., \& Bernstein, E. (2010). A brief motivational interview in a pediatric emergency department, plus 10-day telephone follow-up, increases attempts to quit drinking among youth and young adults who screen positive for problematic drinking. Academic Emergency Medicine, 17, 890-902.

Carey, K. B., Scott-Sheldon, L. A., Elliott, J. C., Garey, L., \& Carey, M. P. (2012). Face-to-face versus computerdelivered alcohol interventions for college drinkers: A meta-analytic review, 1998 to 2010. Clinical Psychology Review, 32(8), 690-703.

Cohen, J (1988). Statistical power analysis for the behavioral sciences. 2nd ed. New York: Lawrence Erlbaum.

Doumas, D. M., Hausheer, R., Esp, S., \& Cuffee, C. (2014). Reducing alcohol use among 9th grade students: 6 month outcomes of a brief, web-based intervention. Journal of Substance Abuse Treatment, 47, 102- 105.

Goti, J., Diaz, R., Serrano, L., Gonzalez, L., Calvo, R., Gual, A., \& Castro, J. (2010). Brief intervention in substanceuse among adolescent psychiatric patients: A randomized controlled trial. European Child \& Adolescent Psychiatry, 19(6), 503-511. doi:10.1007/s00787-009-0060-5

Haines, M., \& Spear, S. F. (1996). Changing the perception of the norm: A strategy to decrease binge drinking among college students. Journal of American College Health, 45(3), 134-140.

Jessor, R., Donovan, J. E., \& Costa, F. M. (1991). Beyond adolescence: Problem behavior and young adult development. New York: Cambridge University Press.

Marlatt, G. A., Baer, J. S., Kivlahan, D. R., Dimeff, L. A., Larimer, M. E., Quigley, L. A., ... Williams, E. (1998). Screening and brief intervention for high-risk college student drinkers: Results from a two-year follow-up assessment. Journal of Consulting and Clinical Psychology, 66, 604-615.

Marlatt, G. A., \& Gordon, J. R. (1985). Relapse prevention: Maintenance strategies in the treatment of addiction behaviors. New York, NY: Guilford Press.

Miller, W. R., \& Rollnick, S. (1991). Motivational interviewing: Preparing people to change addictive behavior. New York, NY: Guilford Press.

Miller, W. R., \& Rollnick, S. (2002). Motivational interviewing: Preparing people for change (2nd ed.). New York, NY: Guilford Press.

Monti, P., Colby, S., Barnett, N., Spirito, A., Rohsenow, D., Myers, M., ... Lewander, W. (1999). Brief intervention for harm reduction with alcohol-positive older adolescents in a hospital emergency department. Journal of Consulting and Clinical Psychology, 67, 989-994.

Moyers, T. B, Martin, T., Manuel, J. K., Miller, W. R., \& Ernst, D. (2010). Motivational Interviewing Treatment Integrity maual (MITI 3.1.1) Center on Alcoholism, Substance Abuse and Addictions: University of New Mexico.

Shaw, D. S., Wagner, E. F., Arnett, J., \& Aber, M. S. (1992). The factor structure of the reckless behavior 
questionnaire. Journal of Youth and Adolescence, 21(3), 305-323.

Sobell, L. C. (1996). Bridging the gap between science and practitioners: The challenge before us. Behavior Therapy, 27, 297-320.

Spirito, A., Barnett, N., Lewander, W., Colby, S., Rohsenow, D., Eaton, C., \& Monti, P. (2001). Risks associated with alcohol-positive status among adolescents in the emergency department: A matched case-control study. Journal of Pediatrics, 139, 694699.

Spirito, A., Monti, P. M., Barnett, N. P., Colby, S. M., Sindelar, H., Rohsenow, D. J., . . Myers, M. (2004). A randomized clinical trial of a brief motivational intervention for alcohol-positive adolescents treated in an emergency department. Journal of Pediatrics, 145(3), 396-402.

Tait, R. J., \& Christensen, H. (2010). Internet-based interventions for young people with problematic substance use: A systematic review. The Medical Journal of Australia, 192(11 Suppl), S15-21.
Walton, M. A., Chermack, S. T., Shope, J. T., Bingham, C. R., Zimmerman, M. A., Blow, F. C., \& Cunningham, R. M. (2010). Effects of a brief intervention for reducing violence and alcohol misuse among adolescents: A randomized controlled trial. Journal of the American Medical Association, 304(5), 527-535.

White, H. R., \& Labouvie, E. W. (1989). Towards the assessment of adolescent problem drinking. Journal of Studies on Alcohol (50), 30-37.

Winters, K. C., Fahnhorst, T., Botzet, A., Lee, S., \& Lalone, B. (2012). Brief intervention for drug-abusing adolescents in a school setting: Outcomes and mediating factors. Journal of Substance Abuse Treatment, 42, 279-288.

Winters, K. C., Lee, S., Botzet, A., Fahnhorst, T., \& Nicholson, A. (2014). One-year outcomes and mediators of a brief intervention for drug abusing adolescents. Psychology of Addictive Behaviors, 28, 464-474. 


\section{Appendices}

\section{Appendix Figure 1}

Consort Figure

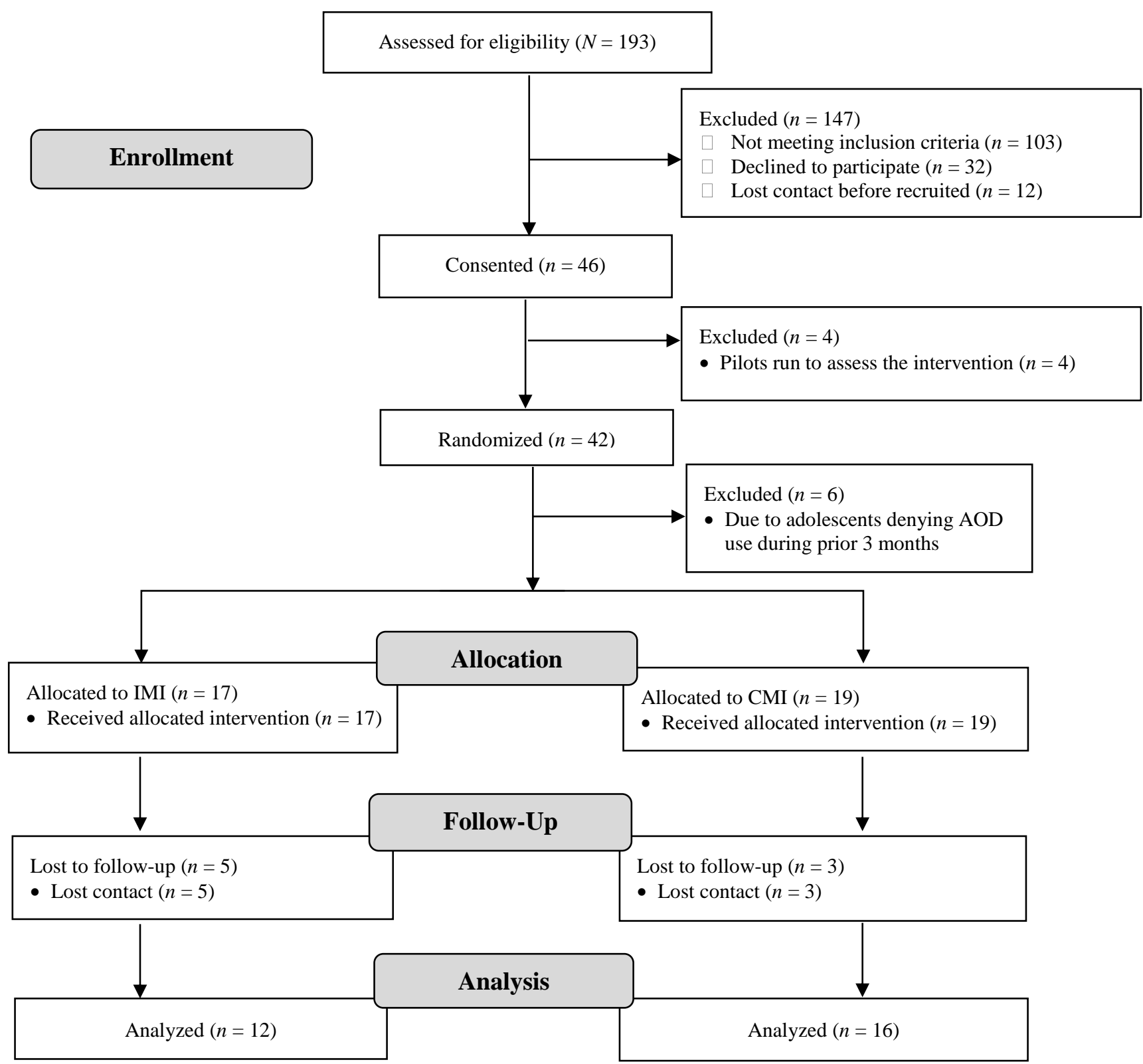

